\title{
K-means Cluster's Classification of Grade of Regional Strong Precipitation Events
}

\author{
Tiangui Xiao Tianyao Shen Fengrong Jing Chao Wang Ding Chen \\ Yaze Wu Yao Huang
}

Chengdu University of Information Technology Atmospheric Sciences Academy, Chengdu 610225, China

\begin{abstract}
Based on the historical precipitation data of the stations in Sichuan Province from 1961 to 2013, 636 heavy precipitation processes were summarized by statistical analysis. First, the daily average of heavy rainfall was selected. Precipitation, rainfall intensity, coverage and duration were used as the index factors of grade division. Secondly, by analyzing the interaction between the index factors, the K-means method was used to analyze the similarity between the distances The heavy precipitation process is divided into five grades by 636 heavy precipitation. Finally, based on the clustering results, a hierarchical model of the operationalized heavy rainfall event in Sichuan Province is established for the risk assessment of the severe precipitation disaster and the forecasting and forecasting of the disaster.
\end{abstract}

\section{Keywords}

Regional; Strong Precipitation; K-means; Cluster

\section{区域性强降水事件等级的 K-means 聚类 划分}

\section{肖天贵 申天瑶 敬枫蓉 王超 陈丁 吴亚泽 黄瑶 \\ 成都信息工程大学大气科学学院, 成都 610225 , 中国}

摘要：基于已信息化的 1961-2013 年四川省各站点逐日的历史降水资料及 636 次强降水过 程, 研究了强降水事件等级划分。首先选取强降水过程的日平均降水量、降水强度、覆盖范 围和持续时间四个指标作为等级划分的指标因子，其次通过对各指标因子之间相互作用的分 析, 提出以 K-means 方法根据各次过程指标间距离的相似性对强降水过程进行聚类分析划 分等级, 将 636 次强降水过程划分为五个等级。基于聚类结果建立四川省可业务化强降水事 件等级模型, 用于强降水灾害的风险评估及灾害的预报预警。

关键词：区域性；强降水； k-means；聚类分析 


\section{1.引言}

在全球气候变化的大背景下, 极 端气象灾害频发, 如暴雨洪涝、干 旱、高温热浪、低温冷害等日益频 繁, 对于地处青藏高原与我国东部平 原的阶梯过渡区的四川盆地, 海拔高 度差大, 地质构造复杂, 受季风影响 较大, 更是我国自然灾害频繁发生且 危害最严重的省份之一。就致灾气象 事件而言, 强降水导致的灾害尤为显 著, 如由强降水引发的洪涝、渍涝、 崩塌、滑坡、泥石流和水土流失等灾 害造成了国民经济和人民生命财产的 巨大损失。对于四川省而言, 每年因 区域性强降水形成的突发性和持续性 暴雨洪水灾害在致灾气象事件中所占 比例高达 $40 \%$, 已成为经济社会可持 续发展的重要制约因素之一, 如 2013 年出现的四川历史上罕见的 “ $7.9 ”$ 强 暴雨（都江堰幸福站过程降水量超过 $1000 \mathrm{~mm}$ ），不仅造成人民财产的损 失, 还使多人失去生命。因此加强对 强降水致灾事件的等级划分及准确的 风险评估的研究是迫切需要的, 这是 防灾减灾的重要依据。

四川盆地的降水呈现出显著的多 尺度变化和极端区域性特征, 强降水 具有出现频率高、年际变化大、影响 大、年内 5-10月均可发生, 以 7-8 月 为盛。强降水引发的灾害的种类和程 度随地形特点的不同而不同, 强降水 致灾过程的复杂性使得灾害的风险评 估成为一项比较艰难的工作, 但是由 于灾害的预报预警的需要, 强降水致 灾的等级划分及风险评估又是必不可 少的, 科学准确的判断强降水事件的 等级, 由此做出灾害的风险评估, 为 当地政府开展防灾工作、确定减灾、 救灾方案、制定灾后救援计划等提供 有力的科技支撑和科学决策依据, 避
免造成重大的损失。评判强降水等级 的指标不只有降水量, 还有其他一些 指标可反映强降水的强度、致灾的可 能性, 通过综合分析讨论, 选取其中 主要的指标进行等级划分, 选取了日 平均降水量、降水强度、覆盖范围以 及持续时间作为描述强降水事件的指 标。目前, 分类方法多种多样, 但由 于各区域的地质构造不同, 引起灾害 的强降水的指标也不同, 所以很难建 立一个统一的分类方法。考虑到各次 降水过程间相似性的大小各不相同, 可将各次过程各指标间的相似性作为 类属划分的准则。在各种聚类算法 中, K-means 算法对大数据有较高的效 率并且是具有可伸缩性的, 能保证局 部收玫, 事先给定聚类数, 根据某种 准则进行快速聚类, 特别是样本分布 呈类内团聚状时, 可以达到较好的聚 类效果。因此本文以 K-means 聚类方 法进行多元分类, 依据各区域的指标 数据库进行分类。分类过程属于无监 督分类的范畴, 希望运用到四川盆地 的区域性强降水的等级划分中，以便 进行四川省区域性强降水致灾事件的 灾害评估的研究, 为当地政府确定减 灾、救灾方案、制定灾害救援计划等 提供有力的支撑和依据。

\section{2.理论方法}

K-means 聚类算法是硬聚类算法 的一种, 也是典型的基于距离的聚类 算法。以欧式距离作为相似度的测 度, 判断各个样本到聚类中心的欧式 距离, 以样本点到聚类中心的欧氏距 离来迭代优化, 根据欧式距离最小来 将样本点重新分配给最近的聚类中 心, 这样将所有的样本划分完之后, 新的聚类中心形成。算法采用误差平 方和准则函数作为聚类准则函数, 一 次聚类结束之后判断误差平方和的大 
小, 若该值变化不大则算法收玫。具 体算法如下:

(1) 设样本即被分类的对象为 $X=\left\{\mathrm{x}_{1}, x_{2}, x_{3} \cdots, x_{n}\right\}$ ，其中 $\mathrm{n}$ 为样本 数, $\mathrm{X}$ 中的每个对象又用 $\mathrm{m}$ 个描述属 性 $A_{1}, A_{2}, \cdots, A_{m}$ (维度)表示, 本文所对 应的样本为 636 次强降水过程, 即 $\mathrm{n}=636, \mathrm{~m}$ 个描述属性为日平均降水量、 降水强度、覆盖范围以及持续时间, 即 $\mathrm{m}=4$;

(2)选取聚类个数 $\mathrm{k}$, 本文要将 636 次强降水过程划分为五个等级, 因此 令 $\mathrm{k}=5$;

(3) 数据样本 $\mathrm{x}_{i}=\left\{x_{i 1}, x_{i 2}, \cdots, x_{i m}\right\}$, $\mathrm{x}_{j}=\left\{x_{j 1}, x_{j 2}, \cdots, x_{j m}\right\}$, 其 中 $\mathrm{x}_{i 1}, x_{i 2}, \cdots, x_{i m}$ 和 $\mathrm{x}_{j 1}, x_{j 2}, \cdots, x_{j m}$ 分别是 样本 $\mathrm{X}_{i}$ 和 $\mathrm{X}_{j}$ 对应的 $\mathrm{m}$ 个描述指标 $A_{1}, A_{2}, \cdots, A_{m}$ 的具体取值。从数据样本 中随机选取 $\mathrm{k}$ 个强降水过程作为聚类 中心, 选取的聚类中心样本的各次过 程间的距离尽可能分开, 以保证聚类 的快速收敛;

(4)样本 $\mathrm{X}_{i}$ 和 $\mathrm{X}_{j}$ 之间的相似度通 常用他们之间的距离 $\mathrm{d}\left(\mathrm{x}_{\mathrm{i}}, x_{j}\right)$ 来表示, 他们之间的距离越小, 样本 $\mathrm{X}_{i}$ 和 $\mathrm{X}_{j}$ 越 相似, 差异度越小; 距离越大, 样本 $\mathrm{X}_{i}$ 和 $\mathrm{X}_{j}$ 越不相似, 差异度越大。计算 原样本数据的每次过程与聚类中心样 本的欧式距离, 判断距离大小, 将每 次过程划分到距离最小的质心类; 欧 式距离:

$$
\mathrm{d}\left(x_{i}, x_{j}\right)=\sqrt{\sum_{k=1}^{d}\left(x_{i k}-x_{j k}\right)^{2}}
$$

(5)计算新的聚类中心，计算各聚 类子集的各指标的平均值作为新的聚 类中心;
(6)使用误差平方和准则函数来评 价聚类性能, 将给定数据集 $\mathrm{X}$, 其中只 包含描述属性, 不包含类别属性。设 $\mathrm{x}$ 包含 $\mathrm{k}(\mathrm{k}=5)$ 个聚类子集, 各个聚类 子集的样本数量为 $\mathrm{n}_{1}, n_{2}, \ldots . . ., n_{k}$, 各个 聚类子集的均值代表点（聚类中心） 分别为 $m_{1}, m_{2}, \cdots, m_{k}$ 。误差平方和准则 函数公式:

$$
E=\sum_{i=1}^{k} \sum_{p \in X}\left\|P-m_{i}\right\|^{2}
$$

判断准则函数收敛: (1)质心的值 变化不大; (2)类内的样本变化不大。

(7)若一次迭代之后不收玫, 则继 续从第 (4) 步进行迭代, 新的聚类中 心取代旧的聚类中心, 直到收敛为 止。

由于 K-means 聚类算法属于硬聚 类算法, 所以存在以下缺点: 一是对 初始聚类中心的选取比较敏感; 二是 对离群点和孤立点较为敏感。

\section{3. 资料及资料处理}

\section{1. 资料选取}

用已信息化的 1961 2013 年四川 省逐日、逐小时历史降水等气象资 料，根据 24 小时降水资料统计从 1961 年到 2013 年的强降水过程, 将降水量 大于等于 $50 \mathrm{~mm}$ 的降水过程, 连续性 的降水记为一次暴雨过程, 记录一次 暴雨过程的发生的时间、范围内的站 点及其降水量, 选取出现的 636 次暴 雨过程。考虑到四川盆地的强降水特 点, 降水的时空分布极不均匀, 夏季 强降水中心比较集中, 可以考虑分 区, 强降水致灾的因子和机理都比较 复杂, 且各种因子间存在相互作用, 本文选取主要的因子忽略次要因子, 选取日平均降水量、降水强度、覆盖 范围和持续时间作为基本指标, 以建 立可业务化的分区域、分等级的四川 
省强降水事件模型。各年强降水次数 统计如下表:

表 1 强降水过程及其年份

\begin{tabular}{|c|c|}
\hline $\begin{array}{l}\text { 强降水过程 } \\
\text { （次/年） }\end{array}$ & 年份 (年) \\
\hline 6 & $2002 、 2006$ \\
\hline 7 & 1976 \\
\hline 8 & 1997、2010 \\
\hline 9 & 1968、1975、1992、1993、2004 \\
\hline 10 & 1972、1982、1986、1994、2008 \\
\hline 11 & $\begin{array}{c}\text { 1965、1970、1995、1996、2003、2005 } \\
\text { 2007、2011 }\end{array}$ \\
\hline 12 & 1964、1987、1988、1991 \\
\hline 13 & $\begin{array}{c}1961 、 1967 、 1973 、 1974 、 1979 、 1990 \\
1998 、 2001\end{array}$ \\
\hline 14 & $\begin{array}{c}1962 、 1966 、 1971 、 1977 、 1978 、 1980 \\
\text { 1981、1989、2013 }\end{array}$ \\
\hline 15 & 1969、1985、1999、2000、2009 \\
\hline 16 & 1983、1984、2012 \\
\hline 17 & 1963 \\
\hline
\end{tabular}

\section{2. 强降水评估指标数据处理}

(1) 日平均降水量指标:

$$
I_{j s}=\frac{1}{n} \sum_{i=1}^{n} p_{i} \quad(\mathrm{i}=1,2, \cdots \cdots, \mathrm{n})
$$

其中, $\mathrm{n}$ 为区域内一次降水过程中降水

量达到暴雨标准的观测站点个数, $P_{j}$ 为其中第 $\mathrm{i}$ 个观测站点在被评估过程中 的总降水量（单位：毫米），表征了 区域的平均降水状况;

(2) 降水强度极值指标:

$$
I_{q d}=\max \left(P_{i}\right) \quad(\mathrm{i}=1, \cdots \cdots, \mathrm{n})
$$

其中, $\max ()$ 为取最大值函数, $P_{j}$ 为 一次降水过程中第 $\mathrm{i}$ 个观测站点在暴雨 过程中的 24 小时观测降水量（单位: 毫米), 这是一个强降水过程中的极 值, 用以表征过程降水最大值, 值越 大, 致灾的可能性越大;

(3) 覆盖范围指标:

$$
I_{f w}=\frac{n}{N}
$$

其中, $\mathrm{N}$ 为区域内观测站点总数（单 位: 个), $\mathrm{n}$ 为发生暴雨过程不重复
(即一次过程多次发生暴雨重复站点 记为一次) 站点数总数, 覆盖范围越 广泛, 说明此次强降水水汽充沛, 可 能是由较大的系统控制, 致灾的可能 性也越大;

(4) 降水持续时间指标:

$$
I_{\text {tim }}=T
$$

其中, $\mathrm{T}$ 为过程持续时间 (单位: 天）, 持续时间较长的强降水过程致 灾的可能性很大, 可能引起暴雨洪 涝、山洪泥石流等灾害现象。

\section{4. 强降水事件等级评估模型建立}

\subsection{K-means 聚类划分等级}

在聚类之前, 先将所获取的数据 资料（1961-2013 年636 次强降水过 程）进行处理，处理方法如下：通过 3.2 的方法, 使用 (3)、（4）、

(5) 和（6）的公式分别计算出各次 强降水过程的评估指标按日平均降水 量、降水强度、覆盖范围和持续时间 的顺序排列, 统计每次强降水过程的 结束时间, 然后与评估指标相对应。 由于数据较多, 因此使用 Fortran 编译 器来处理。规范化的部分数据如下:

表 2 强降水评估指标数据

\begin{tabular}{ccccc}
\hline 时间 & $\begin{array}{c}\text { 日平均降水 } \\
\text { 量 }(\mathrm{mm})\end{array}$ & $\begin{array}{c}\text { 降水强 } \\
\text { 度 }(\mathrm{mm})\end{array}$ & $\begin{array}{c}\text { 覆盖范围 } \\
\left(\begin{array}{c}\text { (站点数 } \\
/ 156)\end{array}\right.\end{array}$ & $\begin{array}{c}\text { 持续时 } \\
\text { 间 } \\
(\text { 天 })\end{array}$ \\
\hline 19610616 & 75.4875 & 129.8 & $5.13 \mathrm{E}-02$ & 1 \\
19610620 & 66.41 & 88.5 & $6.41 \mathrm{E}-02$ & 1 \\
19610628 & 91.30081 & 306 & 0.3846154 & 6 \\
19610706 & 85.88889 & 176.1 & 0.1730769 & 1 \\
19610714 & 70.15285 & 218.8 & 0.3205128 & 3 \\
19610724 & 70.61111 & 128.2 & 0.1153846 & 1 \\
19610728 & 61.08 & 87.8 & $3.21 \mathrm{E}-02$ & 1 \\
\hline
\end{tabular}


续表 2 强降水评估指标数据

\begin{tabular}{ccccc}
\hline 时间 $\begin{array}{c}\text { 日平均降 } \\
\text { 水量 }(\mathrm{mm})\end{array}$ & $\begin{array}{c}\text { 降水强度 } \\
(\mathrm{mm})\end{array}$ & $\begin{array}{c}\text { 覆盖范围 } \\
\text { (站点数 } \\
/ 156)\end{array}$ & $\begin{array}{c}\text { 持续时 } \\
\text { 间 } \\
(\text { 天 })\end{array}$ \\
\hline 19610802 & 87.90833 & 180.6 & $7.69 \mathrm{E}-02$ & 1 \\
19610808 & 51.244 & 140.3 & 0.1538462 & 2 \\
19610814 & 91.26191 & 196 & 0.2564103 & 2 \\
19610820 & 80.11875 & 167.9 & 0.2628205 & 2 \\
19610824 & 71.275 & 102.1 & $5.13 \mathrm{E}-02$ & 1 \\
19610824 & 57.06667 & 63.1 & $3.85 \mathrm{E}-02$ & 1 \\
19620616 & 72.61667 & 97.7 & $7.69 \mathrm{E}-02$ & 1 \\
\hline
\end{tabular}

为解决上述 k-means 算法对离群点 和孤立点的敏感的缺点, 先对要进行 分级的数据进行统计, 先抽出其中的 孤立点, 将剩余的数据进行聚类, 得 到最终的聚类中心，对于之前排除的 孤立点, 计算孤立点与最终聚类中心 的距离, 决定孤立点属于哪一类。孤 立点的统计如 “图 1 强降水的指标因 子的分布” 所示（限于篇幅, 图 1 略）。由以上的统计将如下孤立点抽 出:

表 3 孤立点

\begin{tabular}{lllll}
\hline 结束时间 & 平均降水量 降水强度 & 覆盖范围 & $\begin{array}{l}\text { 持续时 } \\
\text { 间 }\end{array}$ \\
\hline 19610629 & 91.30081 & 306 & 0.3846154 & 6 \\
19930730 & 115.4192 & 524.7 & 0.1602564 & 2 \\
19950824 & 151.7304 & 374.3 & 0.1474359 & 1 \\
19960728 & 96.76 & 410.8 & 0.1858974 & 2 \\
20130701 & 93.45574 & 415.9 & 0.3141026 & 3 \\
\hline
\end{tabular}

再把处理后的数据进行聚类分 级。K-means 聚类的关键在于聚类中心 的选取, K-means 聚类算法是局部收玫 的, 聚类中心选得较好, 收玫速度就 较快, 相反, 聚类中心选得没那么理 想聚类时间就会延长。本文初始聚类 中心的选取如下:
表 4 初始聚类中心

\begin{tabular}{lccccc} 
& 1 级 & 2 级 & 3 级 & 4 级 & 5 级 \\
\hline 平均降雨 & 70.3 & & 90.1 & & 109. \\
量 $/ \mathrm{mm}$ & 3 & 80.49 & 0 & 95.50 & 60 \\
降雨强度 & 88.0 & & 206. & & 311. \\
$/ \mathrm{mm}$ & 0 & 150.30 & 33 & 241.28 & 76 \\
覆盖范围 & 0.06 & 0.10 & 0.18 & 0.26 & 0.34 \\
持续时间 & & & & & \\
$/ \mathrm{d}$ & 1.0 & 2.0 & 2.0 & 3.0 & 4.0 \\
\hline
\end{tabular}

根据四川省区域降水的特点将此 次研究的强降水过程的样本划分为五 类, 从一级到五级表示从轻度到重 度。聚类步骤如下: 先计算 $n$ $(\mathrm{n}=631)$ 个样本与聚类中心 $Z_{K}(\mathrm{k}=1,2,3,4,5)$ 间的欧式距离, 由 公式 (1) 得到样本 $X_{\mathrm{i}}$ 与聚类中心 $Z_{k}$ 间的欧式距离, 然后比较距离大小, 距离越小, 该样本与该聚类中心越相 似, 则可把样本 $X_{\mathrm{i}}$ 划分到第 $\mathrm{k}$ 类, 重 复此过程就将 $\mathrm{n}$ 个样本划分为五类 了, 完成一次聚类过程。例如 1961 年 06 月 16 日的这一次强降水过程, 根据 强降水过程的数据即可算出其与初始 聚类中心 $Z_{1}, Z_{2}, Z_{3}, Z_{4}, Z_{5}$ 的欧式距离 如下:

表 5 与初始聚类中心的欧式距离

\begin{tabular}{lllll}
$d\left(x_{1}, z_{1}\right)$ & $d\left(x_{1}, z_{2}\right.$, & $d\left(x_{1}, z_{3}\right)$ & $d\left(x_{1}, z_{4}\right)$ & $d\left(x_{1}, z_{5}\right)$ \\
42.12 & 21.13 & 77.92 & 116.23 & 185.15 \\
\hline
\end{tabular}

由此可以判断出 $d\left(x_{1}, z_{2}\right)$ 最小, 所以把此次强降水过程划分到第 2 级, 其他样本数据重复此过程, 即可 将所有的强降水过程划分到各个等 级, 最后再统计所以强降水过程, 即 可将样本数据划分为五个等级; 再由 误差平方和准则函数即公式(2)来计算 并判断是否收敛, k-means 聚类方法是 可以保证局部收敛的，设第 0 次聚类 $\mathrm{E}=0$, 其后的聚类依次计算出 $\mathrm{E}$ 值, $\mathrm{E}$ 的值是先递减的, 递减到最小又开始 
增加, 此临界值的地方则可判断聚类 收敛; 若是收玫, 划分结束; 若是不 收敛, 继续进行迭代。迭代方法为将 划分的各类求平均值作为新的聚类中 心, 然后继续从 (1) 开始, 直到收玫 为止。本文所使用的具体的样本数据 在聚类过程当中, 通过两次聚类就达 到了收敛的目的，两次聚类完成后形 成的新的聚类中心与初始聚类中心相 差不大, $E=205351.4$, 新的聚类中心 如下表所示:

\begin{tabular}{lccccc}
\multicolumn{5}{c}{ 表 6 最终聚类中心 } \\
\hline & 1 级 & 2 级 & 3 级 & 4 级 & 5 级 \\
\hline 平均降雨量 & 68.6 & 79.1 & 87.9 & 94.4 & 100. \\
$/ \mathrm{mm}$ & 2 & 1 & 6 & 0 & 14 \\
降 雨 强度 & 98.6 & 147. & 202. & 266. & 349. \\
$/ \mathrm{mm}$ & 0 & 42 & 70 & 79 & 91 \\
覆盖范围 & 0.07 & & & & \\
& 8 & 0.13 & 0.19 & 0.22 & 0.25 \\
持续时间 $/ \mathrm{d}$ & 1.2 & 1.6 & 2.1 & 2.5 & 2.6 \\
\hline
\end{tabular}

\section{2. 划分结果}

通过两次聚类过程, 可将四川省 1963--2013 年间的 636 次强降水过程划 分等级, 然后再统计划分结果, 统计 每一等级的强降水过程及其频数, 具 体每类样本的数量如下表所示:

\begin{tabular}{cccccc}
\multicolumn{8}{c}{ 表7 } & 强降水等级 & & \\
\hline 等级 & 一级 & 二级 & 三级 & 四级 & 五级 \\
频数 & 262 & 215 & 103 & 37 & 14 \\
\hline
\end{tabular}

根据上述的划分则可列出重度暴 雨过程即第五级的详细情况，如下表 所示:

表8 等级划分

\begin{tabular}{ccccc}
\hline 结束时间 & $\begin{array}{c}\text { 平均降水 } \\
\text { 量 }\end{array}$ & 降水强度 & 覆盖范围 & $\begin{array}{c}\text { 持续 } \\
\text { 时间 }\end{array}$ \\
\hline 19610628 & 91.30081 & 306 & 0.3846154 & 6 \\
19640722 & 92.06129 & 310.4 & 0.1538462 & 3 \\
19740818 & 80.71923 & 309.2 & 0.2628205 & 3 \\
19770910 & 93.90513 & 323.4 & 0.1858974 & 2 \\
19830820 & 93.77143 & 314.7 & 0.3141026 & 3 \\
\hline
\end{tabular}

续表8 等级划分

\begin{tabular}{ccccc}
\hline 结束时间 & $\begin{array}{c}\text { 平均降水 } \\
\text { 量 }\end{array}$ & 降水强度 & 覆盖范围 & $\begin{array}{c}\text { 持续 } \\
\text { 时间 }\end{array}$ \\
\hline 19840702 & 103.3762 & 362.3 & 0.2692308 & 1 \\
19840730 & 113.8514 & 379 & 0.1730769 & 2 \\
19880626 & 97.43889 & 315.3 & 0.1153846 & 1 \\
19930730 & 115.4192 & 524.7 & 0.1602564 & 2 \\
19950824 & 151.7304 & 374.3 & 0.1474359 & 1 \\
19960728 & 96.76 & 410.8 & 0.1858974 & 2 \\
19970816 & 83.8775 & 311.7 & 0.1858974 & 2 \\
19980708 & 85.10271 & 356.6 & 0.3653846 & 4 \\
20070708 & 81.55833 & 325.5 & 0.1217949 & 2 \\
20130700 & 93.45574 & 415.9 & 0.3141026 & 3 \\
20130720 & 81.75957 & 358.7 & 0.25 & 3 \\
\hline
\end{tabular}

\section{3. 强降水事件等级确定}

文章已经把 1961-2013 年的历史强 降水过程划分为了五个等级, 建立了 一个有效的聚类中心，对于单个的强 降水过程可根据此聚类中心来划分。 若有一个强降水过程, 先由 3.2 的一系 列公式计算出强降水评估指标，然后 利用公式 (2) 计算出此次强降水过程 与表 5 的最终聚类中心的欧式距离, 然后判断此次强降水过程属于哪一 级。具体步骤如下:

设一次强降水过程为 $X=\left\{x_{1}, x_{2}, x_{3}, x_{4}\right\}$, 最终聚类中心为 $\mathrm{Z}$ ，计 算 $\mathrm{X}_{i}(\mathrm{i}=1,2,3,4)$ 与 $Z_{k}$ $(\mathrm{k}=1,2,3,4,5)$ 的欧式距离, 公式为 $\mathrm{d}\left(X, Z_{k}\right)=\sqrt{\sum_{i=1}^{d}\left(X_{i}-Z_{k i}\right)^{2}}$ 。根据欧式距 离的大小来划分 $\mathrm{X}$ 的等级。

\section{5. 总结}

本文选取了四个指标对强降水等 级进行评判, 意在建立一个具有普遍 
性能应用到四川省的强降水等级评 估, 这与其他分级结果有一些出入, 对于有几个强降水过程分级稍有不 同，但大体一致，说明说明通过 Kmeans 聚类的方法对强降水过程分级有 一定的意义。运用 K-means 聚类方法 对强降水事件进行等级划分, 能使聚 类中心达到最优，方法简单可行，能 快速的进行聚类, 且聚类效果可行, 综合考虑了强降水事件的四个指标因 子对其进行聚类, 等级划分合理, 是 强降水致灾风险评估的根据, 因此能 用到实际业务中去, 为当地政府开展 防灾工作、确定减灾、救灾方案、制 定灾后救援计划等提供有力的科技支 撑和科学决策依据, 避免造成重大的 损失。

基于可拓模型的四川暴雨灾害风 险评估模型的建立是非常有意义的工 作, 对于气象业务有重要的促进作 用。文中选取致灾因子、孕灾环境、 承灾体、防灾减灾能力作为一级评估 因子, 并将单站暴雨过程总量、暴雨 过程平均量、最大日暴雨量、地形、 坡度、人口密度、地均 GDP、耕地比 例、人均 GDP、公路里程作为二级评 估因子, 并通过区域暴雨特征分析提 出的高原站和非高原站的暴雨灾害分 级、通过对数正态分布函数获得重现 期作为致灾因子等级评估标准等思 路, 对业务应用都是有实践意义的。 但由于暴雨灾害影响的复杂性以及致 灾因子资料收集的困难，风险评估模 型建立的恰当还需进一步研究。

\section{致谢}

大学生创新创业训练计划项目 (CX2015017) 、中国气象局西南区 域气象中心重大科研项目（西南区域 014-5)、国家自然科学基金 （91337215，41575066）、国家科技 支撑计划（2015BAC03B05）、公益性
行业 (气象) 科研专项 （GYHY201406015）、国家重点基础 研究发展计划（2013CB733206）。

\section{参考文献}

[1] 章国才.气象灾害风险评估与区划方 法.气象出版社.2010.

[2] 王小玲,翟盘茂.1957-2004 年中国不 同强度级别降水的变化趋势特征. 热 带气象学报,2008,24(5):459-466.

[3] 王千,王成, 冯振远, 叶金风.K-means 聚类算法研究综述. 电子设计工 程.2012,20(7):21-24.

[4] 宋连春,肖风劲,叶殿秀.气象灾害影 响及风险评估理论与实践.气象出版 社.2012.

[5] 吴夙慧,成颖,郑彦宁,潘云涛.Kmeans 算法研究综述*. 现代图书情 报技术.2011(5):28-35.

[6] 黄蹈,刘胜辉,谭艳娜. 基于 K-means 聚类算法的研究.计算机技术与发 展.2011,21(7):54-57.

[7] 刘合香. 模糊数学理论及其应用.科 学出版社.2012,8. 